\title{
A novel small peptide as an epidermal growth factor receptor targeting ligand for nanodelivery in vitro
}

\author{
Cui-yan Han ${ }^{1,2}$ \\ Li-ling Yue ${ }^{2}$ \\ Ling-yu Tai ${ }^{\prime}$ \\ Li Zhou ${ }^{2}$ \\ Xue-yan Li ${ }^{2}$ \\ Gui-hua Xing ${ }^{2}$ \\ Xing-gang Yang' \\ Ming-shuang Sun' \\ Wei-san Pan' \\ 'School of Pharmacy, Shenyang \\ Pharmaceutical University, Shenyang, \\ People's Republic of China; ${ }^{2}$ Qiqihar \\ Medical University, Qiqihar, People's \\ Republic of China
}

Correspondence: Wei-San Pan School of Pharmacy, Shenyang Pharmaceutical University, 103 Wenhua Road, Shenyang, I 100 16, People's Republic of China Tel +8624239863 I3 Fax +86 2423953241 Email pwstfzy@।63.com
This article was published in the following Dove Press journal:

International Journal of Nanomedicine

18 April 2013

Number of times this article has been viewed

\begin{abstract}
The epidermal growth factor receptor (EGFR) serves an important function in the proliferation of tumors in humans and is an effective target for the treatment of cancer. In this paper, we studied the targeting characteristics of small peptides (AEYLR, EYINQ, and PDYQQD) that were derived from three major autophosphorylation sites of the EGFR $\mathrm{C}$-terminus domain in vitro. These small peptides were labeled with fluorescein isothiocyanate (FITC) and used the peptide LARLLT as a positive control, which bound to putative EGFR selected from a virtual peptide library by computer-aided design, and the independent peptide RALEL as a negative control. Analyses with flow cytometry and an internalization assay using NCI-H1299 and K562 with high EGFR and no EGFR expression, respectively, indicated that FITC-AEYLR had high EGFR targeting activity. Biotin-AEYLR that was specifically bound to human EGFR proteins demonstrated a high affinity for human non-small-cell lung tumors. We found that AEYLR peptide-conjugated, nanostructured lipid carriers enhanced specific cellular uptake in vitro during a process that was apparently mediated by tumor cells with high-expression EGFR. Analysis of the MTT assay indicated that the AEYLR peptide did not significantly stimulate or inhibit the growth activity of the cells. These findings suggest that, when mediated by EGFR, AEYLR may be a potentially safe and efficient delivery ligand for targeted chemotherapy, radiotherapy, and gene therapy.
\end{abstract}

Keywords: EGFR, small peptide, tumor targeting, lung cancer, NLC

\section{Introduction}

The discovery of suitable ligands that bind to cancer cells is important for drug delivery specifically targeted to tumors. ${ }^{1}$ Monoclonal antibodies and fragments that serve as ligands have specific targets; however, they are limited in terms of their immunogenicity. ${ }^{2-6}$ Natural ligands have strong mitogenic and neoangiogenic activities. Currently, small peptides are pursued as targeting moieties because of their small size, low immunogenicity, and their ability to be incorporated into certain delivery vectors. ${ }^{7-12}$

The epidermal growth factor receptor (EGFR) is a transmembrane protein on the cell surface that is overexpressed in a wide variety of human cancers. EGFR is an effective tumor-specific target because of its significant functions in tumor cell growth, differentiation, and migration. ${ }^{13-15}$ EGFR-targeted small molecule peptides such as YHWYGYTPQNVI have been successfully identified using phage display library screening; ${ }^{8}$ by contrast, the peptide LARLLT has been generated using computerassisted design (CAD). ${ }^{10}$ These peptides can be conjugated to the surfaces of liposomes that are then delivered selectively to tumors by the specific and efficient binding of these peptides to cancer cells that express high levels of EGFR. ${ }^{10,16}$ 
In this study, we focused on determining whether small peptides are targeted from the amino acid sequence of the receptor C-terminus. EGFR has three major autophosphorylation sites (Y1068, Y1148, and Y1173) in the C terminus. ${ }^{17}$ We designed 5- to 6-mer synthetic peptides that were derived from the amino acid sequences of these sites $\left(E Y^{1068} I N Q, P D Y^{1148} \mathrm{QQD}\right.$, and $A E Y{ }^{1173} L R$, respectively). We then studied the ability of these peptides and NYQQN ${ }^{18}$ to target EGFR in vitro, using LARLLT $^{10}$ as a positive control and RALEL (AEY ${ }^{1173} \mathrm{LR}$, substituted and scrambled) as a negative control.

\section{Materials and methods}

\section{Antibodies and reagents}

EGFR (D38B1) XP ${ }^{\circledR}$ Rabbit monoclonal antibody was obtained from Cell Signaling Technology (Danvers, MA, USA). Alexa Flour 488-labeled goat anti-rabbit immunoglobulin $\mathrm{G}(\mathrm{IgG})$ and horseradish peroxidase (HRP)-streptavidin were obtained from Beyotime Inc (Haimen, People's Republic of China). Purified EGFR derived from human carcinoma A431 cells and polyoxyethylene (40) stearate were both obtained from Sigma (Shanghai, People's Republic of China). Goat anti-rabbit IgG, HRP-IgG, and FITC-avidin were obtained from Boster Biotechnology Co, Ltd (Fremont, CA, USA). Purified soybean oil 788 and egg lecithin (EPC) were obtained from Lipoid GmbH (Ludwigshafen, Germany). 1,2-distearoyl-sn-glycero-3-phosphoethanolamine- $N$ [biotinyl(polyethylene glycol)-2000] (DSPE- PEG $_{2000}$-Biotin) and 1,2-distearoyl-sn-glycero-3-phosphoethanolamine$N$-[carboxy (polyethylene glycol)-2000] (DSPE-PEG ${ }_{2000^{-}}$ $\mathrm{COOH}$ ) were purchased from Avanti Polar Lipids, Inc (Alabaster, AL, USA). Other reagents used were of an analytical grade or higher.

\section{Cell culture and peptide synthesis}

The human non-small-cell lung cancer cell line NCI-H1299 and chronic myeloid original leukemia cell line K562 were purchased from the Cell Bank of the Chinese Academy of Sciences (Beijing, People's Republic of China). The cells were cultured in Roswell Park Memorial Institute (RPMI)1640 medium (NCI-H1299) or Iscove's Modified Dulbecco's Medium (IMDM) (K562) and were supplemented with $10 \%$ heat-inactivated fetal bovine serum (FBS), penicillin $(100 \mathrm{U} / \mathrm{mL})$, and streptomycin $(100 \mathrm{U} / \mathrm{mL})$ at $37^{\circ} \mathrm{C}$ in a humidified atmosphere containing $5 \% \mathrm{CO}_{2}$. Peptides (AEYLR, EYINQ, PDYQQD, NYQQN, LARLLT, and RALEL) had a purity greater than $98 \%$ and were synthesized and labeled (FITC, biotin) by TeraBIO GZ Co, Ltd (Guangzhou, People's Republic of China).

\section{Evaluation of the expression of EGFR}

NCI-H1299 cells were washed with phosphate-buffered solution (PBS) ( $\mathrm{pH} 7.4$ ), detached with $0.2 \%$ ethylenediaminetetraacetic acid (EDTA), and counted. Since K562 cells are suspension cells, they were directly counted. The cells were fixed in $4 \%$ paraformaldehyde for 10 minutes at $37^{\circ} \mathrm{C}$ and then chilled on ice for 1 minute. The cells were rinsed twice with $2 \mathrm{~mL}$ of PBS-bovine serum albumin (BSA) (PBS, $5 \mathrm{mg} / \mathrm{mL} \mathrm{BSA}$ ) and blocked with PBS-BSA, then incubated with an anti-EGFR antibody (1:100) for 1 hour at room temperature, followed by incubation with Alexa Flour 488labeled secondary antibody (1:200) for 30 minutes at room temperature. ${ }^{19}$ Ten thousand cells per sample were analyzed using flow cytometry (BD, San Jose, CA, USA). Only viable cells were gated for fluorescence analysis.

\section{Binding assays}

The binding affinities between the peptides and the NCIH1299 or K562 cells were measured using flow cytometry. The cells were treated using the methods described above. FITC-labeled peptides $(6 \mu \mathrm{M})$ were incubated with $4 \times 10^{5}$ NCI-H1299 or K562 cells in PBS-BSA in tubes for 1 hour at room temperature. Cells were then centrifuged at $1500 \mathrm{rpm}$ for 5 minutes, after which the supernatant was discarded. Subsequently, the cells were rinsed three times with $0.7 \mathrm{~mL}$ PBS. Stained cells were analyzed using flow cytometry. Only viable cells were gated for fluorescence analysis.

\section{Internalization assay}

NCI-H1299 cells $\left(1 \times 10^{5}\right.$ cells $)$ were cultured overnight on cover slips in a 24-well plate. When the cells had grown to $70 \%-80 \%$ confluence, the medium was replaced with $300 \mu \mathrm{L}$ of fresh, serum-free medium that contained $10 \mu \mathrm{M}$ FITC-labeled peptides. The plate was incubated for 3 hours at $37^{\circ} \mathrm{C}$. The slices were fixed with $4 \%$ paraformaldehyde for 20 minutes and washed by PBS. Afterward, images were taken using a fluorescence microscope (Olympus Corporation, Tokyo, Japan). Since K562 cells are suspension cells and could not be cultured on the cover slips, they were directly incubated with the FITC-labeled peptides. The remainder of the procedure was repeated as described above.

\section{Immunohistochemistry of selected peptides}

Paraffin sections of human non-small-cell lung cancer tissue were provided by the pathologic diagnosis center at Qiqihar Medical University (Qiqihar, People's Republic of China). Immunohistochemistry was conducted overnight 
using EGFR antibodies at a dilution of 1:100 and $10 \mu \mathrm{M}$ biotin-AEYLR or biotin-RALEL at $4^{\circ} \mathrm{C}$. HRP-IgG and HRP-streptavidin were added the following day. The resulting solutions were incubated at room temperature for 1 hour and then stained with diaminobenzidine (Maixin Bio, Jinan City, People's Republic of China). After rinsing with PBS, the tissue slides were visualized and imaged using a fluorescence microscope.

\section{Characterization of selected peptides via enzyme-linked immunosorbent assay (ELISA)}

Up to $0.5 \mu \mathrm{g}$ of human EGFR or BSA in $50 \mu \mathrm{L}$ of $0.1 \mathrm{M}$ $\mathrm{NaHCO}_{3}$ was used to coat a 96-well plate overnight. The following day the wells were washed three times with PBS and blocked with $200 \mu \mathrm{L}$ of PBS-BSA (PBS, $10 \mathrm{mg} / \mathrm{mL}$ BSA). Biotin peptides of approximately $25 \mu \mathrm{M}$ were diluted in $100 \mu \mathrm{L}$ of PBS-BSA and added to each well. After incubating at $4{ }^{\circ} \mathrm{C}$ for 3 hours, the wells were again washed three times with PBST (PBS, $0.5 \%$ Tween-20). ${ }^{8}$ The bound peptides were incubated with HRP-conjugated streptavidin at room temperature for 1 hour and were developed with color using an HRP substrate solution (Beyotime Inc). The color development was stopped after the addition of $2 \mathrm{M} \mathrm{H}_{2} \mathrm{SO}_{4}$ and was then measured at $450 \mathrm{~nm}$ using an ELISA reader (Safire, ${ }^{2}$ Tecan Group Ltd, Männedorf, Swizterland). An unrelated peptide was also included in the experiment as a control.

\section{MTT assay}

Cell growth or inhibition was measured by MTT (SigmaAldrich, St Louis, MO, USA) assay. ${ }^{20}$ Briefly, $5 \times 10^{3}$ NCI-H1299 cells were seeded overnight in each well of a 96-well plate in RPMI-1640 medium, each containing 10\% FBS. Cells were cultured in $100 \mu \mathrm{L}$ of fresh medium that contained various concentrations of AEYLR or RALEL for 48 hours. The MTT solution $(20 \mu \mathrm{L}, 5 \mathrm{mg} / \mathrm{mL}$ in PBS $)$ was added to each well and the plate was incubated for 4 hours at $37^{\circ} \mathrm{C}$. The solution was removed and $200 \mu \mathrm{L}$ of dimethyl sulfoxide (DMSO) was added to each well. After 10 minutes of vibration mixing, the optical density (OD) at $570 \mathrm{~nm}$ was measured using an ELISA reader.

\section{Fluorescence microscopy and flow cytometry of nanostructured lipid carriers (NLCs) incubated with $\mathrm{NCl}-\mathrm{HI} 299$ cells}

The NLCs were prepared using melt emulsification followed by ultrasonography (Branson Sonifier* S-250D Digital
Ultrasonic Cell Disruptor/Homogenizer; Thermo Fisher Scientific, Waltham, MA, USA). ${ }^{21}$ The mixture included glyceryl monostearate, lecithin, soybean oil, DSPE-PEG ${ }_{2000}-\mathrm{COOH}$, and polyoxyethylene (40) stearate. AEYLR and RALEL were conjugated with NLCs using the method of Almiñana et al. ${ }^{22}$ During preparation of the FITC-NLC, the DSPE-PEG ${ }_{2000^{-}}$ Biotin was added as a lipid and the NLCs were prepared as previously described. The FITC was coupled with the NLCs through the biotin-avidin system. The internalization analysis of the NLCs was performed by incubating the NCIH1299 cells with FITC-NLC, FITC-AEYLR-NLC, or FITCRALEL-NLC. The cells were then seeded in a 12-well plate at a density of $2 \times 10^{5}$ cells per well and incubated overnight at $37^{\circ} \mathrm{C}$. The growth medium was removed and the cells were washed twice with PBS. The FITC-labeled NLCs with serumfree medium was added. The cells were incubated for 3 hours at $37^{\circ} \mathrm{C}$ and were subsequently fixed in $4 \%$ paraformaldehyde for 20 minutes. The fluorescence in the cells was observed using an inverted fluorescence microscope. Afterward, all of the cells were harvested by trypsinization and were washed three times in PBS. The cell-associated fluorescence was quantitatively determined using flow cytometry in which 10,000 events were counted. Only viable cells were gated for fluorescence analysis.

\section{Results}

\section{Binding activities of the small peptides of the EGFR C-terminus}

To test the feasibility of the peptides as ligands for specific molecular recognition, the FITC-labeled peptides (AEYLR, EYINQ, PDYQQD, NYQQN, LARLLT, and RALEL) and monoclonal antibodies were used to detect the expression of EGFR on NCI-H1299 and K562 cell surfaces. Flow cytometry was carried out using Alexa Flour 488-labeled antibodies. Our analysis revealed that the NCI-H1299 cells expressed substantial levels of EGFR (Figure 1A) while the K562 cells did not (Figure 1B).

The binding activities between the peptides and the NCI-H1299 or K562 cells were measured using flow cytometry. The sequence of the binding activities between the NCI-H1299 and FITC-labeled peptides was AEYLR > LARLLT $>$ PDYQQD $>$ NYQQN $>$ EYINQ $>$ RALEL, as shown in Figure 1C and D. The FITC-AEYLR peptide had the highest binding activities among the peptides we surveyed. The binding rates of FITC-AEYLR and FITC-LARLLT to NCI-H1299 were $93.09 \% \pm 3.42 \%$ and $73.81 \% \pm 1.16 \%$, respectively. The binding rates of all peptides to K562 cells were less than 15\% (Figure 1D and E). Our results suggest 


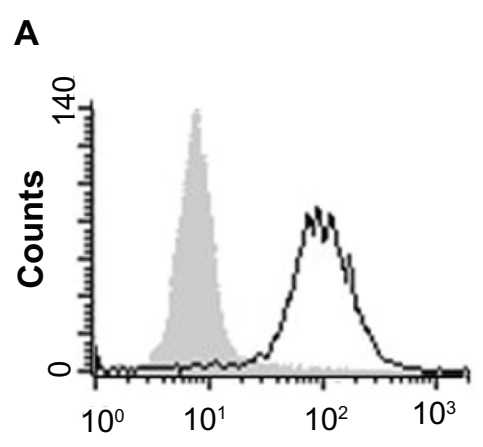

FL-1

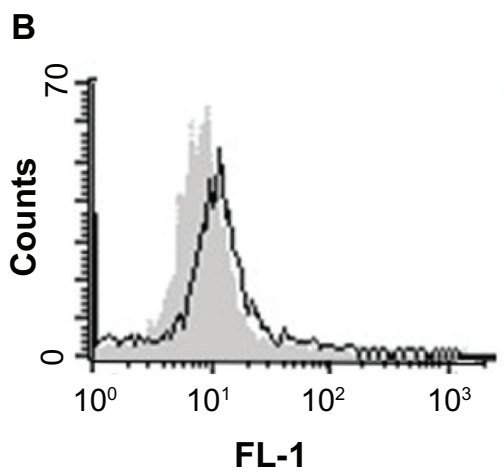

C

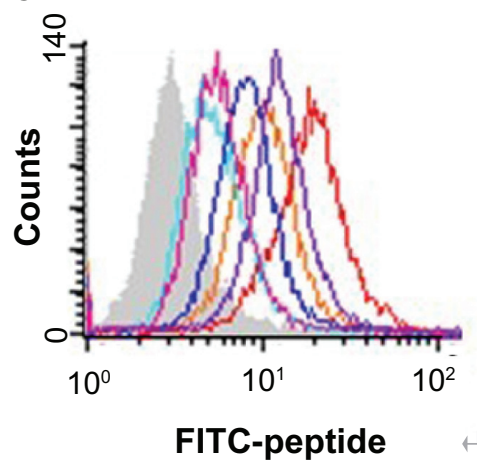

E

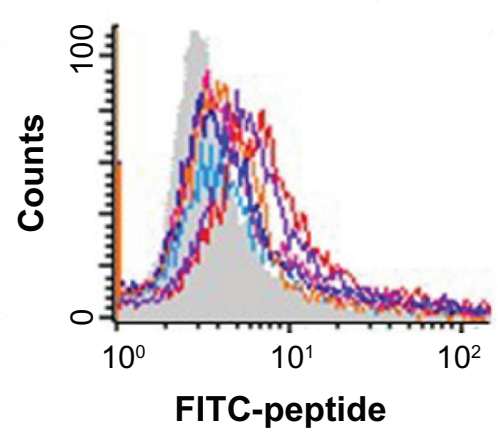

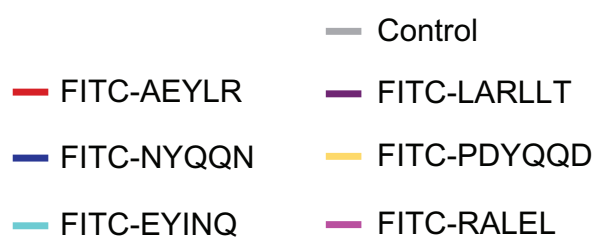

$\mathrm{NCl}-\mathrm{H} 1299$

K562

Figure I Binding activities of the small peptides. The expression of EGFR on (A) NCl-HI299 and (B) K562 was confirmed by flow cytometry. The gray background indicates non-treatment and the black lines indicate the results obtained for the EGFR antibody treatment. (C) $\mathrm{NCl}-\mathrm{HI} 299$ cells binding with FITC-labeled peptide using flow cytometry. (D) Comparison of binding percentages of FITC-labeled peptides with NCI-HI299 and K562 cells using flow cytometry. (E) K562 cells binding with FITC-labeled peptide by flow cytometry.

Note: Data shown are presented as mean \pm SEM and were obtained from three independent experiments.

Abbreviations: EGFR, epidermal growth factor receptor; FITC, fluorescein isothiocyanate; SEM, standard error of the mean.

that AEYLR can recognize cells expressing high levels of EGFR and that our methods can select high affinity ligands for those cells.

\section{Internalization assay}

To examine whether the peptides may be internalized into EGFR-expressing cells, the NCI-H1299 and K562 cells were incubated with the FITC-labeled peptides. As expected, significant fluorescence was observed after incubating the FITC-AEYLR and FITC-LARLLT peptides with the NCI$\mathrm{H} 1299$ cells at $37^{\circ} \mathrm{C}$ for 3 hours. Minimal fluorescence was observed after incubating the peptides with the K562 cells (Figure 2). These results indicate that FITC-AEYLR was efficiently taken up by cells expressing high levels of EGFR and are consistent with the results from flow cytometry.

\section{Immunohistochemistry}

The specific binding activity of the AEYLR peptide with human non-small-cell lung tumors was evaluated using immunohistochemistry. The EGFR expression in human non-small-cell lung cancer paraffin tissues was detected by monoclonal antibodies (Figure 3A). Biotin-labeled peptides were used to evaluate the targeting of non-small-cell lung cancer tissues. Compared with the negative control peptide and PBS, treatment with biotin-AEYLR yielded a more intense color in the cytoplasm of the cells (Figure 3B-D).

Immunohistochemical experiments on frozen sections of non-small-cell lung cancer tissues were also conducted. Fresh tissues were obtained in patients undergoing surgery according to the guidelines of Qiqihar Medical University. After freezing the tissue, $7 \mu \mathrm{M}$ thick tissue sections were prepared using a cryostat and fixed in 4\% paraformaldehyde for 20 minutes. The tissues were then blocked with PBS-BSA (PBS, $20 \mathrm{mg} / \mathrm{mL}$ BSA) at room temperature for 30 minutes and incubated with a solution of $10 \mu \mathrm{M}$ FITC-labeled peptides in PBS at room temperature for 1 hour. The fluorescence detection results from these frozen sections were similar to those acquired using paraffin sections (data not shown).

\section{Characterization of the selected peptide}

To confirm the specificity of the AEYLR peptide's binding to EGFR, AEYLR was labeled with biotin to evaluate 

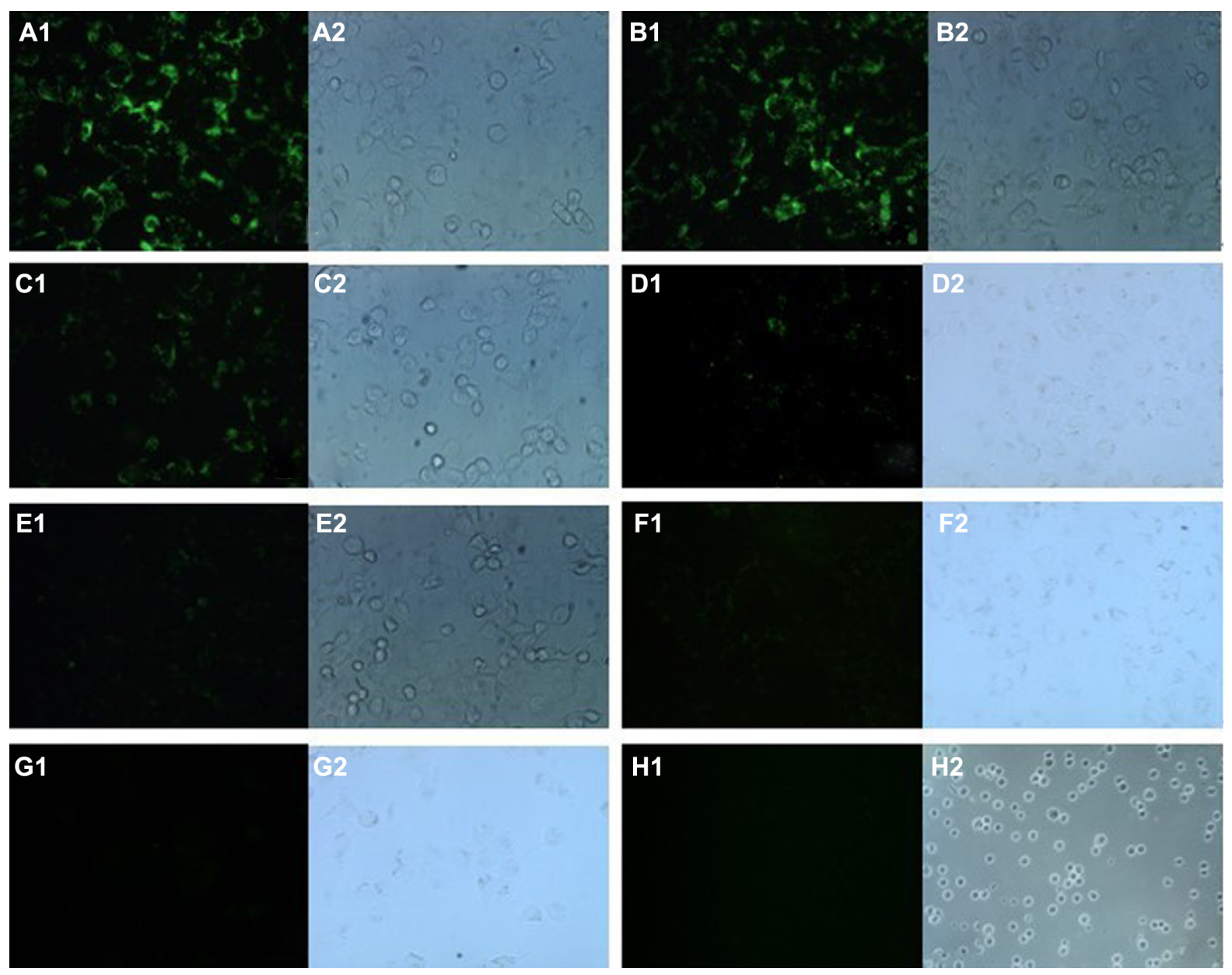

Figure 2 Internalization of peptides. NCI-HI299 cells were incubated with $10 \mu$ M (AI) FITC-AEYLR, (BI) FITC-LARLLT, (CI) FITC-PDYQQD, (DI) FITC-NYQQN, (EI) FITC-EYINQ, (FI) FITC-RALEL, and (GI) FITC at $37^{\circ} \mathrm{C}$ for 3 hours; K562 cells were incubated with $10 \mu M$ FITC-peptide (HI) under the same conditions. Phase contrast micrographs of the same fields (A2 to $\mathbf{H 2}$ ).

Notes: Images were taken using a fluorescence microscope. Magnification: $\times 200$.

Abbreviation: FITC, fluorescein isothiocyanate.

and compare its binding affinities for human EGFR and BSA by use of a colorimetric peroxidase assay. Figure 4A reveals that significantly more biotin-AEYLR was bound to EGFR than to BSA. The OD of biotin-AEYLR bound to EGFR was five times higher than the OD of biotin-AEYLR bound to BSA. No significant binding was found between biotin-AEYLR and BSA, or between biotin-RALEL and EGFR or BSA. These data further support specific binding of AEYLR to EGFR.

\section{Biologic activity of AEYLR}

To evaluate whether the AEYLR peptide inhibited or stimulated cell proliferation, we carried out a standard MTT assay using NCI-H1299 cells. The survival rates of cells exposed to AEYLR and RALEL at concentrations ranging from $0.5 \mu \mathrm{M}$ to $50 \mu \mathrm{M}$ were close to $100 \%$ (Figure 4B). The MTT assay indicated that AEYLR might be safely targeting the ligand because it did not significantly stimulate or inhibit cell growth.

\section{Fluorescence microscopy and flow cytometric analysis of the NLCs incubated with the $\mathrm{NCl}-\mathrm{HI} 299$ cells}

The mean particle size of a FITC-AEYLR-NLC was $123.30 \mathrm{~nm} \pm 2.52 \mathrm{~nm}$ (polydispersity index $0.336 \pm 0.010$ ). The controls, FITC-NLC and FITC-RALEL-NLC, had mean particle sizes of $115.62 \mathrm{~nm} \pm 1.98 \mathrm{~nm}$ (polydispersity index $0.228 \pm 0.011$ ) and $126.90 \mathrm{~nm} \pm 2.17 \mathrm{~nm}$ (polydispersity index $0.393 \pm 0.012$ ), respectively, which indicates that the average ligand coupling efficiency was $3.63 \% \pm 2.93 \%$. The function of AEYLR as an EGFR-targeting ligand conjugated onto the surface of NLCs was determined in vitro. Figure 4C-E summarize the results obtained using flow cytometry and fluorescence microscopy after incubating 

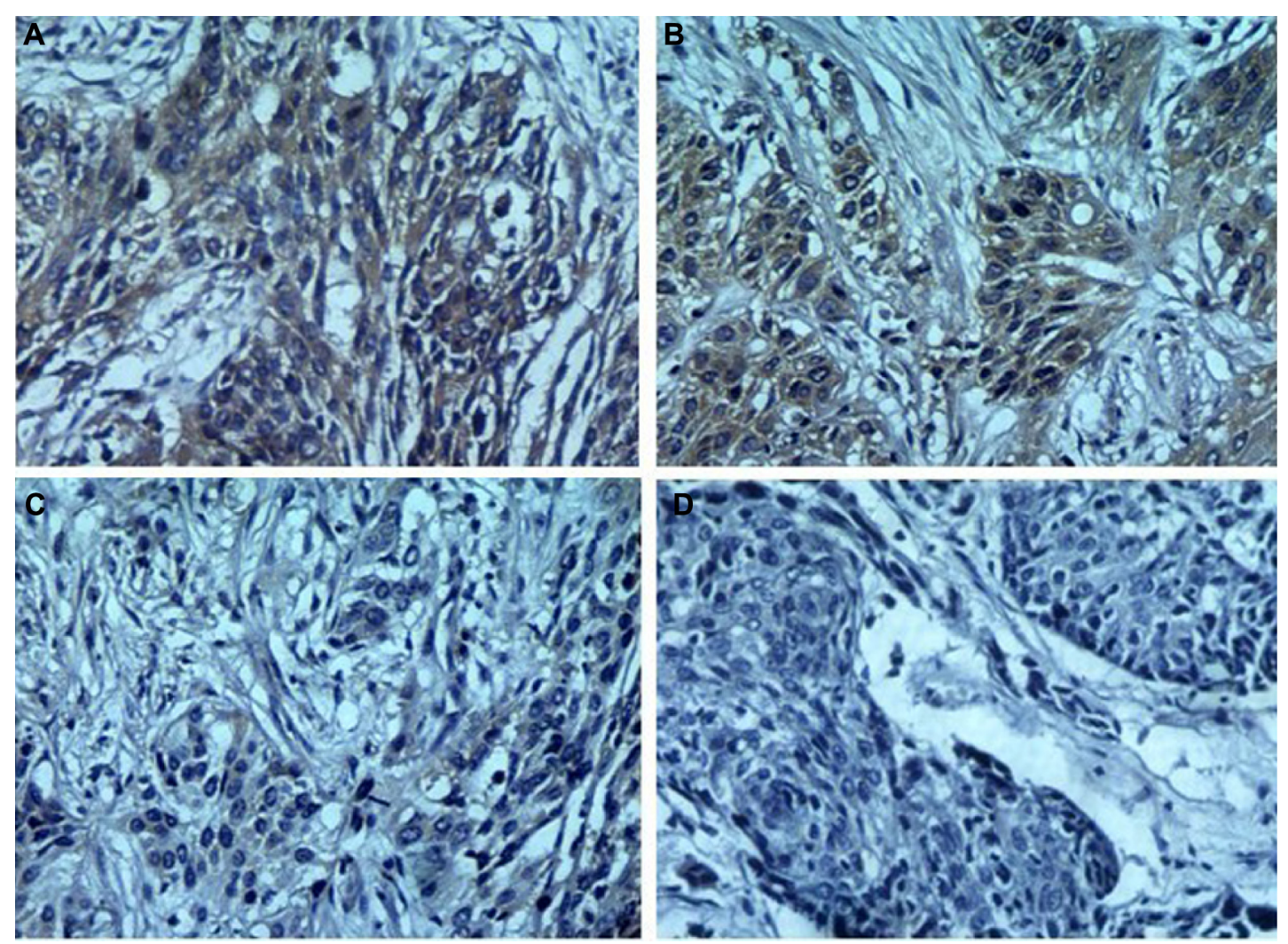

Figure 3 Immunohistochemical results of human non-small-cell lung cancer tissue. (A) EGFR expressed on human non-small-cell lung cancer tissue identified by EGFR mAb (I:I00). (B) Biotin-AEYLR (I0 $M \mathrm{M})$ co-localized with human non-small-cell lung cancer tissue, but (C) the control peptide biotin-RALEL did not. (D) The same result as (C) were observed for PBS

Note: Magnification: $\times 200$.

Abbreviations: EGFR, epidermal growth factor receptor; mAb, monoclonal antibody; PBS, phosphate-buffered solution.

the NCI-H1299 cells with the FITC-labeled NLCs. FITCAEYLR-NLC showed strong fluorescence, whereas FITCNLC and FITC-RALEL-NLC showed minimal fluorescence after being incubated with inherently EGFR-overexpressing NCI-H1299 cells. No significant difference in the fluorescence intensity was detected among the cells treated with FITC-RALEL-NLC or FITC-NLC.

According to the experiments, AEYLR could mediate the active targeting of the NLCs. The higher cytotoxicity of the AEYLR-NLC may be the result of stronger internalization. We concluded that AEYLR-NLC designed to bind specifically to EGFR may be used to deliver chemotherapeutic drugs to tumor cells in vivo.

\section{Discussion}

Small peptides are attractive target ligands because of their size. Screening of phage display libraries, ${ }^{8,23} \mathrm{CAD},{ }^{10}$ hydropathic complementarity ${ }^{24}$ hydropathic analysis, and comparative sequence/structure analysis ${ }^{7}$ have been used to design small peptide ligands. In this study, we demonstrated different methods of screening for small molecule peptide ligands. Flk-1(A-3) is a mouse monoclonal antivascular endothelial growth factor receptor (VEGFR)-2 antibody raised against amino acids $1158-1345$ that map the C-terminus of the VEGFR-2 (Flk-1). ${ }^{25}$ Liu et al found that Flk-1(A-3)-conjugated NLCs enhanced specific cellular uptake. ${ }^{25}$ We believe that the small peptide of the receptor $\mathrm{C}$-terminus has certain targeting abilities. A peptide sequence was also designed based on the findings of Abe et al ${ }^{26} \mathrm{Y} 1068$, Y1148, and Y1173 are the three major autophosphorylation sites of EGFR, ${ }^{18}$ while Y992, Y1045, and Y1086 are minor sites. ${ }^{27-29} \mathrm{Abe}$ et al designed 5- to 7-mer synthetic peptides originating from the amino acid sequences of Y992, Y1068, Y1148, and Y1173 and investigated the effects of the synthetic peptides on the phosphorylation of EGFR. ${ }^{26}$ The results indicated that the small peptides of major autophosphorylation sites exhibited different degrees of inhibition, while small peptides of minor autophosphorylation sites did not. Furthermore, the peptide NYQQN, which was modified from $\mathrm{DY}^{1148} \mathrm{QQD}$, a small peptide from a major autophosphorylation site, may improve the inhibitory potencies and was not affected by the concentration of adenosine 


\section{A}

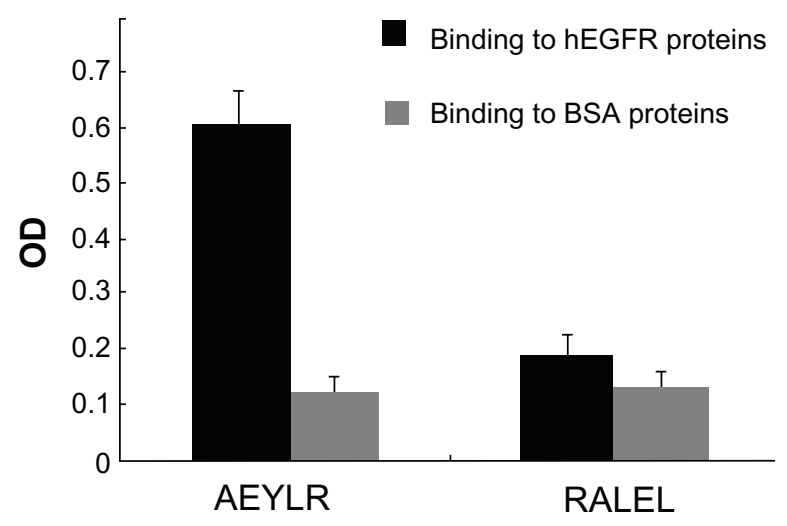

\section{B}

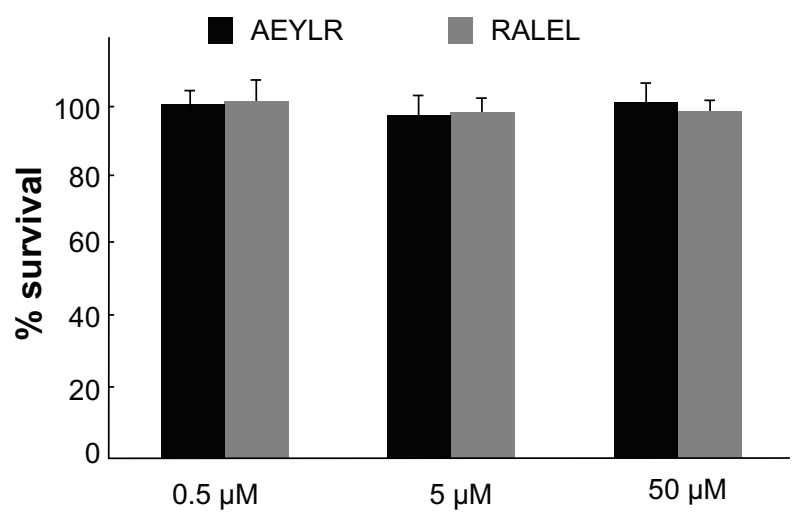

C
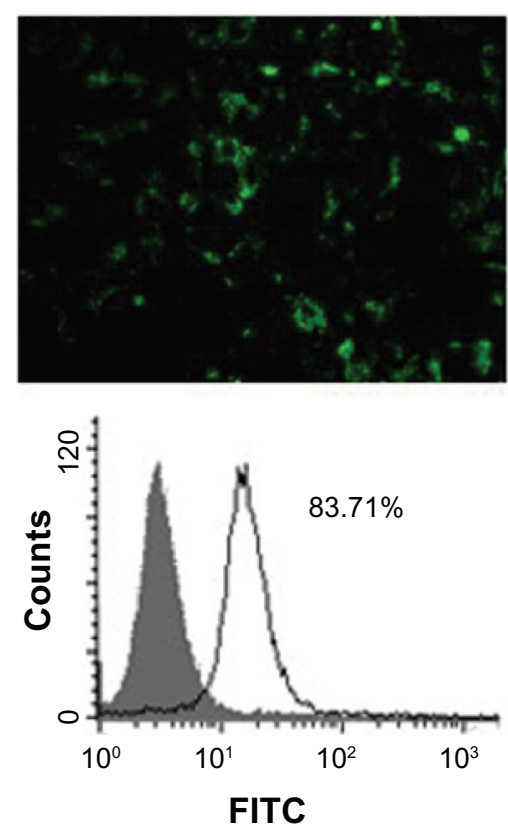

D
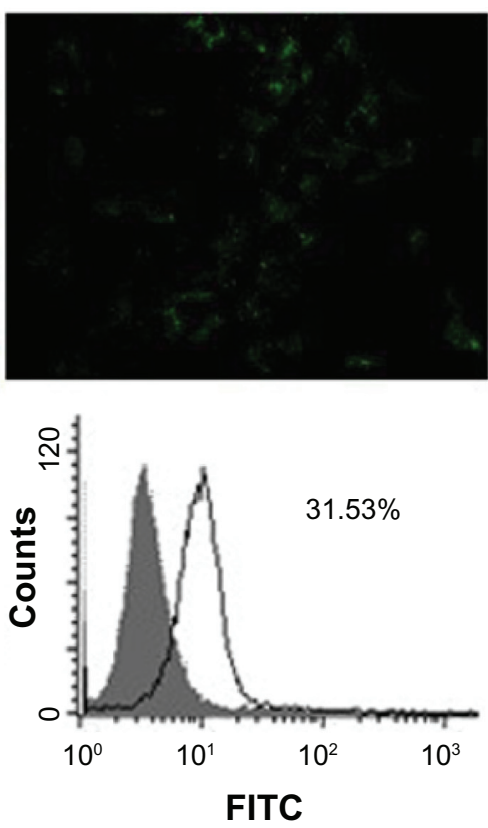

E
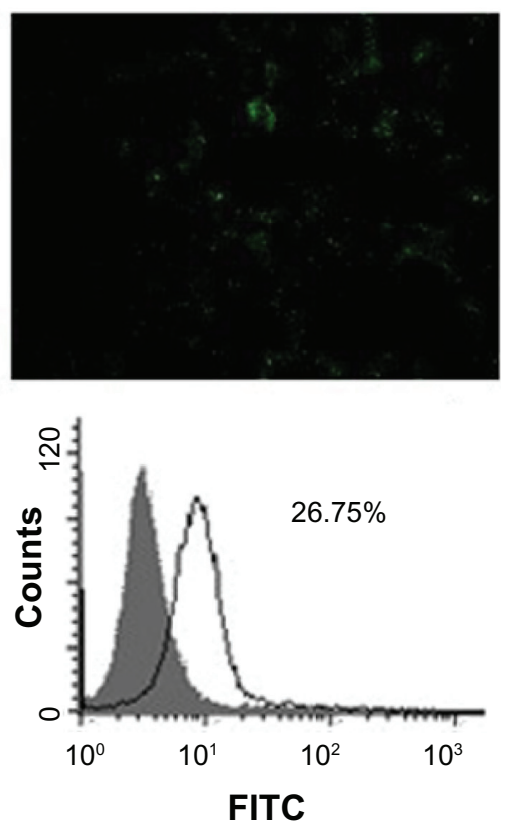

Figure 4 (A) Biotin-AEYLR-bound or biotin-RALEL-bound hEGFR proteins or BSA proteins. (B) Activity of AEYLR and RALEL peptides on NCI-HI299 cell growth was studied via MTT assay. Fluorescence microscopy images and flow cytometry of (C) FITC-AEYLR-NLC, (D) FITC-RALEL-NLC, and (E) FITC-NLC in the presence of NCIHI 299 cells after incubation for 3 hours at $37^{\circ} \mathrm{C}$.

Abbreviations: BSA, bovine serum albumin; FITC, fluorescein isothiocyanate; hEGFR, human epidermal growth factor receptor; NLC, nanostructured lipid carrier; OD, optical density.

triphosphate (ATP). ${ }^{18,26}$ According to Abe et al's ${ }^{26}$ experimental results, we focused on amino acid sequences from the major autophosphorylation sites of EGFR. Specifically, we designed five amino acid sequences from the Y1068 and Y1173 sites (EY $\left.{ }^{1068} \mathrm{INQ}, \mathrm{AEY}{ }^{1173} \mathrm{LR}\right)$, investigated the targeting of NYQQN, and designed six amino acid sequences from the Y1148 site (PDY $\left.{ }^{1148} \mathrm{QQD}\right)$. RALEL is the arranged sequence of the disrupted $A E Y^{1173} \mathrm{LR}$ in which tyrosine residue $(\mathrm{Y})$ was replaced by leucine $(\mathrm{L})$.

Flow cytometry has been widely used in a variety of aptamer screening procedures. ${ }^{30,31}$ Lee et al selected two stabilin-2-specific, FITC-conjugated synthetic peptides, CRTLTVRKC and CIKRGGKLC, using phage display technology, and found that CRTLTVRKC bound to stabilin2-expressing cell lines, while CIKRGGKLC did not. ${ }^{11}$ This finding suggests that the identification of a peptide ligand by FITC labeling is feasible. We labeled our peptides with FITC, incubated them with cells that highly expressed EGFR to be used for flow cytometric analysis, and then carried out several tests. Our experiments indicated that the binding rates of the peptides with targeted cells varied with cell numbers and changes in experimental conditions; however, 
the overall trend was consistent. FITC-AEYLR showed a higher targeting activity than the other peptides designed, and internalization assay experiments further confirmed this finding. Since we believe that evidence of the AEYLR acting as a targeting ligand was only based on flow cytometric analyses and internalization, our assay experiments may be insufficient, and the selection of a suitable peptide as a positive control was necessary. LARLLT, which is a ligand that specifically binds to EGFR receptors, has been identified using a CAD approach. ${ }^{10}$ The LARLLT peptide-conjugated liposomes could bind to the highly expressing EGFR cells (NCI-H1299) and efficiently enter the cells via endocytosis. ${ }^{10}$ Our flow cytometric analysis showed that the binding rate of the FITC-AEYLR peptide with the NCI-H1299 cells was higher than that of the FITC-LARLLT peptide. Strong fluorescence from two peptides was detected in our internalization assay experiments. The experimental results indicated that AEYLR had targeting activities toward EGFR. AEYLR could specifically bind to both the human EGFR protein and the human non-small-cell lung tumors that expressed EGFR. We prepared the NLCs by conjugating AEYLR as a targeting element to evaluate whether it was able to deliver a variety of therapeutic agents directly to the cells as a target ligand.

It is well known that epidermal growth factor (EGF) is the natural ligand of EGFR, but it will stimulate cell proliferation when used as targeting ligand of drug carriers; therefore, small peptides derived from EGF would not be safe due to the risk of stimulating cell proliferation. Small peptides derived from the C-terminal of EGFR show inhibition effects of autophosphorylation, so if we employ these small peptides as targeting ligands of drug carriers, synergistic effects will be obtained, and it will be of great significance in increasing therapeutic effects and decreasing adverse effects in tumor treatment and in improving the quality of life of the patients. The present research was developed based on the objectives declared above, and our results have showed that the small peptide AEYLR, derived from the C-terminal of EGFR, did not stimulate cell proliferation and is safe for use as a targeting ligand of drug carriers.

\section{Conclusion}

This is the first study to identify a small peptide derived from the C-terminus of EGFR for targeted delivery. We demonstrated that the selected peptide was conjugated to NLCs and enhanced the binding and in vitro entry into overexpressing EGFR cells. Further investigations of peptide conjugations to other delivery systems in various preclinical models will be required. The findings in this paper provide new directions for the study of small peptide ligand selection for the delivery of cancer therapies.

\section{Acknowledgment}

This research was supported by the National Science Foundation (No 30973656).

\section{Disclosure}

The authors report no conflicts of interest in this work.

\section{References}

1. Talekar M, Kendall J, Denny W, Garg S. Targeting of nanoparticles in cancer: drug delivery and diagnostics. Anticancer Drugs. 2011;22(10): 949-962.

2. Lukyanov AN, Elbayoumi TA, Chakilam AR, Torchilin VP. Tumortargeted liposomes: doxorubicin-loaded longcirculationg liposomes modified with anti-cancer antibody. J Control Release. 2004;100(1): 135-144.

3. Mamot C, Ritschard R, Küng W, Park JW, Herrmann R, Rochlitz CF. EGFR-targeted immunoliposomes derived from the monoclonal antibody EMD72000 mediate specific and efficient drug delivery to a variety of colorectal cancer cells. J Drug Target. 2006;14(4):215-223.

4. Zhou Y, Drummond DC, Zou H, et al. Impact of single-chain Fv antibody fragment affinity on nanoparticle targeting of epidermal growth factor receptor-expressing tumor cells. J Mol Biol. 2007;371(4):934-947.

5. Nechansky A, Kircheis R. Immunogenicity of therapeutics: a matter of efficacy and safety. Expert Opin Drug Discov. 2010;5(11): 1067-1079

6. Choudhary S, Mathew M, Verma RS. Therapeutic potential of anticancer immunotoxins. Drug Discov Today. 2011;16(11-12):495-503.

7. Wu X, Zhao R, Li Z, et al. A novel small peptide as a targeting ligand for receptor tyrosine kinase Tie2. Biochem Biophys Res Commun. 2004;315(4):1004-1010.

8. Li Z, Zhao R, Wu X, et al. Identification and characterization of a novel peptide ligand of epidermal growth factor receptor for targeted delivery of therapeutics. FASEB J. 2005;19(14):1978-1985.

9. Chen Z, Deng J, Zhao Y, Tao T. Cyclic RGD peptide-modified liposomal drug delivery system: enhanced cellular uptake in vitro and improved pharmacokinetics in rats. Int J Nanomedicine. 2012;7:3803-3811.

10. Song S, Liu D, Peng J, et al. Novel peptide ligand directs liposomes toward EGFR high-expressing cancer cells in vitro and in vivo. FASEB J. 2009;23(5):1396-1404.

11. Lee GY, Kim JH, Oh GT, Lee BH, Kwon IC, Kim IS. Molecular targeting of atherosclerotic plaques by a stabilin-2-specific peptide ligand. J Control Release. 2011;155(2):211-217.

12. Zhang Y, Zhang H, Wang X, Wang J, Zhang X, Zhang Q. The eradication of breast cancer and cancer stem cells using octreotide modified paclitaxel active targeting micelles and salinomycin passive targeting micelles. Biomaterials. 2012;33(2):679-691.

13. Hackel PO, Zwick E, Prenzel N, Ullrich A. Epidermal growth factor receptors: critical mediators of multiple receptor pathways. Curr Opin Cell Biol. 1999;11(2):184-189.

14. McCawley LJ, O'Brien P, Hudson LG. Overexpression of the epidermal growth factor receptor contributes to enhanced ligand-mediated motility in keratinocyte cell lines. Endocrinology. 1997;138(1):121-127.

15. Blume-Jensen P, Hunter T. Oncogenic kinase signalling. Nature. 2001;411(6385):355-365.

16. Song S, Liu D, Peng J, et al. Peptide ligand-mediated liposome distribution and targeting to EGFR expressing tumor in vivo. Int $J$ Pharm. 2008;363(1-2):155-161.

17. Downward J, Parker P, Waterfield MD. Autophosphorylation sites on the epidermal growth factor receptor. Nature. 1984;311(5985): $483-485$. 
18. Abe M, Kuroda Y, Hirose M, et al. Inhibition of autophosphorylation of epidermal growthfactor receptor by a small peptide not employing an ATP-competitive mechanism. Biopolymers. 2008;89(1):40-51.

19. Kibria G, Hatakeyama H, Ohga N, Hida K, Harashima H. Dual-ligand modification of PEGylated liposomes shows better cell selectivity and efficient gene delivery. J Control Release. 2011;153(2):141-148.

20. Hansen MB, Nielsen SE, Berg K. Re-examination and further development of a precise and rapid dye method for measuring cell growth/cell kill. J Immunol Methods. 1989;119(2):203-210.

21. Zhang X, Gan Y, Gan L, Nie S, Pan W. PEGylated nanostructured lipid carriers loadedwith 10-hydroxycamptothecin: an efficient carrier with enhanced anti-tumour effects against lung cancer. J Pharm Pharmacol. 2008;60(8):1077-1087.

22. Almiñana N, Polo D, Rodríguez L, Reig F. Biodistribution study of doxorubicin encapsulated in liposomes: influence of peptide coating and lipid composition. Prep Biochem Biotechnol. 2004;34(1):77-96.

23. Askoxylakis V, Garcia-Boy R, Rana S, et al. A new peptide ligand for targeting human carbonic anhydrase IX, identified through the phage display technology. PLoS One. 2010;5(12):e15962.

24. Campa MJ, Kuan CT, O’Connor-McCourt MD, Bigner DD, Patz EF Jr. Design of a novel small peptide targeted against a tumor-specific receptor. Biochem Biophys Res Commun. 2000;275(2):631-636.

25. Liu D, Liu F, Liu Z, Wang L, Zhang N. Tumor specific delivery and therapy by double-targeted nanostructured lipid carriers with antiVEGFR-2 antibody. Mol Pharm. 2011;8(6):2291-2301.
26. Abe M, Kuroda Y, Hirose M, Watanabe Y, Nakano M, Handa T. Inhibition of autophosphorylation of epidermal growth factor receptor by small peptides in vitro. Br J Pharmacol. 2006;147(4):402-411.

27. Walton GM, Chen WS, Rosenfeld MG, Gill GN. Analysis of deletions of the carboxyl terminus of the epidermal growth factor receptor reveals self-phosphorylation at tyrosine 992 and enhanced in vivo tyrosine phosphorylation of cell substrates. J Biol Chem. 1990;265(3): 1750-1754.

28. Levkowitz G, Waterman H, Ettenberg SA, et al. Ubiquitin ligase activity and tyrosine phosphorylation underlie suppression of growth factor signaling by c-Cbl/Sli-1. Mol Cell. 1999;4(6):1029-1040.

29. Margolis BL, Lax I, Kris R, et al. All autophosphorylation sites of epidermal growth factor (EGF) receptor and HER2/neu are located in their carboxyl-terminal tails. Identification of a novel site in EGF receptor. J Biol Chem. 1989;264(18):10667-10671.

30. Liu Z, Duan JH, Song YM, et al. Novel HER2 aptamer selectively delivers cytotoxic drug to HER2-positive breast cancer cells in vitro. J Transl Med. 2012;10:148.

31. Chen F, Hu Y, Li D, Chen H, Zhang XL. CS-SELEX generates highaffinity ssDNA aptamers as molecular probes for hepatitis $\mathrm{C}$ virus envelope glycoprotein E2. PLoS One. 2009;4(12):e8142.
International Journal of Nanomedicine

\section{Publish your work in this journal}

The International Journal of Nanomedicine is an international, peerreviewed journal focusing on the application of nanotechnology in diagnostics, therapeutics, and drug delivery systems throughout the biomedical field. This journal is indexed on PubMed Central, MedLine, CAS, SciSearch $®$, Current Contents ${ } /$ Clinical Medicine,

\section{Dovepress}

Journal Citation Reports/Science Edition, EMBase, Scopus and the Elsevier Bibliographic databases. The manuscript management system is completely online and includes a very quick and fair peer-review system, which is all easy to use. Visit http://www.dovepress.com/ testimonials.php to read real quotes from published authors. 